TITLE:

\title{
Improved approximation bounds for the Student-Project Allocation problem with preferences over projects
}

\section{$\operatorname{AUTHOR(S):~}$}

Iwama, Kazuo; Miyazaki, Shuichi; Yanagisawa, Hiroki

\section{CITATION:}

Iwama, Kazuo ...[et al]. Improved approximation bounds for the Student-Project

Allocation problem with preferences over projects. Journal of Discrete Algorithms 2012, 13: 59-66

ISSUE DATE:

2012-05

URL:

http://hdl.handle.net/2433/155464

\section{RIGHT:}

(c) 2012 Elsevier B.V.; This is not the published version. Please cite only the published version.; この論文は出版社版でありません。引用の際に は出版社版をご確認ご利用ください。 


\title{
Improved Approximation Bounds for the Student-Project Allocation Problem with Preferences over Projects
}

\author{
Kazuo Iwama ${ }^{\mathrm{a}}$, Shuichi Miyazaki ${ }^{\mathrm{b}, *}$, Hiroki Yanagisawa ${ }^{\mathrm{c}}$ \\ ${ }^{a}$ Graduate School of Informatics, Kyoto University, Yoshida-Honmachi, Sakyo-ku \\ Kyoto-shi, Kyoto 606-8501, Japan \\ ${ }^{b}$ Academic Center for Computing and Media Studies, Kyoto University, \\ Yoshida-Honmachi, Sakyo-ku Kyoto-shi, Kyoto 606-8501, Japan \\ ${ }^{c}$ IBM Research - Tokyo, 1623-14 Shimotsuruma, Yamato-shi, Kanagawa 242-8502, \\ Japan.
}

\begin{abstract}
Manlove and O'Malley [8] proposed the Student-Project Allocation Problem with Preferences over Projects (SPA-P). They proved that the problem of finding a maximum stable matching in SPA-P is APX-hard and gave a polynomial-time 2-approximation algorithm. In this paper, we give an improved upper bound of 1.5 and a lower bound of 21/19 (> 1.1052).
\end{abstract}

Keywords: The student project allocation problem, Stable matching, NP-hardness, Approximation algorithm, Approximation ratio

\section{Introduction}

Assignment problems based on the preferences of participants, which originated from the famous Hospitals/Residents problem $(H R)$ [3], are important

\footnotetext{
${ }^{\text {t }}$ A preliminary version of this paper was presented at the 8th Annual Conference on Theory and Applications of Models of Computation, TAMC 2011. This work was supported by KAKENHI 22240001 and 20700009.

${ }^{*}$ Corresponding author

Email addresses: iwama@kuis.kyoto-u.ac.jp (Kazuo Iwama), shuichi@media.kyoto-u.ac.jp (Shuichi Miyazaki), yanagis@jp.ibm.com (Hiroki Yanagisawa)
} 
almost everywhere, such as in education systems where students must be allocated to elementary schools or university students to projects. In the university case, each student may have preferences over certain research projects supervised by professors and usually there is an upper bound on the number of students each project can accept. Our basic goal is to find a "stable" allocation where no students (or projects or professors if they also have preferences over students) can complain of unfairness. This notion of stability was first introduced by Gale and Shapley in the context of the famous Stable Marriage problem in 1962 [2].

The Student-Project Allocation problem (SPA) is a typical formulation of this kind of problem originally described by Abraham, Irving, and Manlove [1]. The participants here are students, projects, and lecturers. Each project is offered by a single lecturer, though one lecturer may offer multiple projects. Each project and each lecturer has a capacity. Students have preferences over projects, and lecturers have preferences over students. Our goal is to find a stable matching between students and projects satisfying all of the capacity constraints for projects and lecturers. They proved that all stable matchings for a single instance have the same size, and proposed linear-time algorithms to find one [1].

Manlove and O'Malley [8] proposed a variant of SPA, called SPA with Preferences over Projects $(S P A-P)$, where lecturers have preferences over projects they offer rather than preferences over students. In contrast to SPA, they pointed out that the sizes of stable matchings may differ, and proved that the problem of finding a maximum stable matching in SPA-P, denoted $M A X-S P A-P$, is APX-hard. They also presented a polynomial-time 2-approximation algorithm. Specifically, they provided a polynomial-time algorithm that finds a stable matching, and proved that any two stable matchings differ in size by at most a factor of two.

Our Contributions. In this paper, we improve both the upper and lower bounds on the approximation ratio for MAX-SPA-P. We give an upper bound of 1.5 and a lower bound of 21/19 ( $>1.1052)$ (under the condition that $\mathrm{P}$ $\neq \mathrm{NP}$ ). For the upper bound, we modify Manlove and O'Malley's algorithm SPA-P-APPROX [8] using Király's idea [7] for the approximation algorithm to find a maximum stable matching in a variant of the stable marriage problem $(M A X-S M T I)$. We also show that our analysis is tight. For the lower bound, we give a gap-preserving reduction from (a variant of) MAX-SMTI. Our reduction also gives a lower bound of 1.25 under the Unique Games 
Conjecture.

\section{Preliminaries}

Here we give a formal definition of SPA-P and MAX-SPA-P, derived directly from the literature [8]. An instance $I$ of SPA-P consists of a set $S$ of students, a set $P$ of projects, and a set $L$ of lecturers. Each lecturer $\ell_{k} \in L$ offers a subset $P_{k}$ of projects. Each project is offered by exactly one lecturer, i.e., $P_{k_{1}} \cap P_{k_{2}}=\emptyset$ if $k_{1} \neq k_{2}$. Each student $s_{i} \in S$ has an acceptable set of projects, denoted $A_{i}$, and has a strict order on $A_{i}$ according to preferences. Each lecturer $\ell_{k}$ also has a strict order on $P_{k}$ according to preferences. Also, each project $p_{j}$ and each lecturer $\ell_{k}$ has a positive integer, called a capacity, denoted $c_{j}$ and $d_{k}$, respectively.

An assignment $M$ is a subset of $S \times P$ where $\left(s_{i}, p_{j}\right) \in M$ implies $p_{j} \in A_{i}$. Let $\left(s_{i}, p_{j}\right) \in M$ and $\ell_{k}$ be the lecturer who offers $p_{j}$. Then we say that $s_{i}$ is assigned to $p_{j}$ in $M$, and $p_{j}$ is assigned $s_{i}$ in $M$. We also say that $s_{i}$ is assigned to $\ell_{k}$ in $M$ and $\ell_{k}$ is assigned $s_{i}$ in $M$.

For $s \in S$, let $M(s)$ be the set of projects to which $s$ is assigned in $M$. For $r \in P \cup L$, let $M(r)$ be the set of students assigned to $r$ in $M$. If $M\left(s_{i}\right)=\emptyset$, we say that the student $s_{i}$ is unassigned in $M$, otherwise $s_{i}$ is assigned in $M$. We say that the project $p_{j}$ is under-subscribed, full, or over-subscribed with respect to $M$ according to whether $\left|M\left(p_{j}\right)\right|<c_{j},\left|M\left(p_{j}\right)\right|=c_{j}$, or $\left|M\left(p_{j}\right)\right|>c_{j}$, respectively, under $M$. If $\left|M\left(p_{j}\right)\right|>0$, we say that $p_{j}$ is non-empty, otherwise, it is empty. Corresponding definitions apply to each lecturer $\ell$.

A matching $M$ is an assignment such that $\left|M\left(s_{i}\right)\right| \leq 1$ for each $s_{i}$, $\left|M\left(p_{j}\right)\right| \leq c_{j}$ for each $p_{j}$, and $\left|M\left(\ell_{k}\right)\right| \leq d_{k}$ for each $\ell_{k}$. For a matching $M$, if $\left|M\left(s_{i}\right)\right|=1$, we may use $M\left(s_{i}\right)$ to denote the unique project to which $s_{i}$ is assigned. The size of a matching $M$, denoted $|M|$, is the number of students assigned in $M$.

Given a matching $M$, a (student, project) pair $\left(s_{i}, p_{j}\right)$ blocks $M$, or is a blocking pair for $M$, if the following three conditions are met:

1. $p_{j} \in A_{i}$.

2. Either $s_{i}$ is unassigned or $s_{i}$ prefers $p_{j}$ to $M\left(s_{i}\right)$.

3. $p_{j}$ is under-subscribed and either

(a) $s_{i} \in M\left(\ell_{k}\right)$ and $\ell_{k}$ prefers $p_{j}$ to $M\left(s_{i}\right)$, or

(b) $s_{i} \notin M\left(\ell_{k}\right)$ and $\ell_{k}$ is under-subscribed, or

(c) $s_{i} \notin M\left(\ell_{k}\right), \ell_{k}$ is full, and $\ell_{k}$ prefers $p_{j}$ to $\ell_{k}$ 's worst non-empty 
project,

where $\ell_{k}$ is the lecturer who offers $p_{j}$.

Given a matching $M$, a coalition is a set of students $\left\{s_{i_{0}}, s_{i_{1}}, \ldots, s_{i_{r-1}}\right\}$ for some $r \geq 2$ such that each $s_{i_{j}}$ is assigned in $M$ and prefers $M\left(s_{i_{j+1}}\right)$ to $M\left(s_{i_{j}}\right)$, where $j+1$ is taken modulo $r$. A matching that has no blocking pair nor coalition is stable. Refer to [8] for the validity of this definition of stability. SPA-P is the problem of finding a stable matching, and MAX-SPA-P is the problem of finding a maximum stable matching.

We say that $A$ is an $r$-approximation algorithm if it satisfies $O P T(I) / A(I) \leq$ $r$ for all instances $I$, where $O P T(I)$ and $A(I)$ are the sizes of the optimal and the algorithm's solutions for $I$, respectively.

\section{Approximability}

\subsection{Algorithm SPA-P-APPROX-PROMOTION}

Manlove and O'Malley's algorithm SPA-P-APPROX [8] proceeds as follows. First, all students are unassigned. Any student $(s)$ who has non-empty preference list applies to the top project $(p)$ on the current list of $s$. If the lecturer $(\ell)$ who offers $p$ has no incentive to accept $s$ for $p$, then $s$ is rejected. When rejected, $s$ deletes $p$ from the list. Otherwise, $(s, p)$ is added to the current matching. If, as a result, $\ell$ becomes over-subscribed, $\ell$ rejects a student from $\ell$ 's worst non-empty project to satisfy the capacity constraint. This continues until there is no unassigned student whose preference list is non-empty. Manlove and O'Malley proved that the obtained matching is stable.

We extend SPA-P-APPROX using Király's idea [7]. During the execution of our algorithm SPA-P-APPROX-PROMOTION, each student has one of two states, "unpromoted" or "promoted". At the beginning, all of the students are unpromoted. The application sequence is unchanged. When a student $(s)$ becomes unassigned with her preference list exhausted, $s$ is promoted. When promoted, $s$ returns to her original preference list (i.e., all of the previous deletions are canceled) and starts a second sequence of applications from the top of her list. For the decision rule for acceptance or rejection by the lecturers, they will prefer promoted students to unpromoted students within the same project. The formal description of SPA-P-APPROX-PROMOTION is given as Algorithm 1.

When a student $s_{i}$ applies to a project $p_{j}$ but is instantly rejected, we say that $p_{j}$ rejects $s_{i}$ and $s_{i}$ is rejected by $p_{j}$. Similarly, when a student $s_{i}$ being 
assigned to $p_{j}$ is rejected (due to another student's application), we say that $p_{j}$ rejects $s_{i}$ and $s_{i}$ is rejected by $p_{j}$.

\subsection{Correctness}

It is straightforward to show that SPA-P-APPROX-PROMOTION outputs a matching in polynomial time. We will now show that the output matching $M$ is stable. We first prove two useful lemmas:

Lemma 3.1. Suppose that, during the execution of SPA-P-APPROX-PROMOTION, a project $p_{a}$ rejected a promoted student. Then (i) after that point, no student can be accepted to $p_{a}$, and (ii) no unpromoted student can be assigned to $p_{a}$ in $M$.

ProOF. Suppose that a promoted student $s$ is rejected by $p_{a}$. Let $\ell_{k}$ be the lecturer who offers $p_{a}$. It is easy to see that just after this rejection, no unpromoted student can be assigned to $p_{a}$. We show that after that point, if a student $s^{\prime}$ applies to $p_{a}$ when there is no unpromoted student assigned to $p_{a}$, then $s^{\prime}$ must be rejected. It is easy to see that the lemma follows by using this fact inductively.

Note that just after this rejection, either (1) $p_{a}$ is full or (2) $p_{a}$ is undersubscribed and $\ell_{k}$ is full. We consider Case (2) first. Since $p_{a}$ is undersubscribed but $s$ was rejected by $p_{a}$, just before this rejection $p_{a}$ must be $\ell_{k}$ 's worst non-empty project or even worse than $\ell_{k}$ 's worst non-empty project. Then after this rejection, $p_{a}$ remains $\ell_{k}$ 's worst non-empty project or worse than that. Note that now $\ell_{k}$ remains full until the end of the execution. Then after this point, when any student applies to $p_{a}$, only Cases A (line 10) or B (line 16) of the algorithm can apply. Since there is no unpromoted student in $M\left(p_{a}\right), s^{\prime}$ must be rejected.

In Case (1), if $p_{a}$ is still full when $s^{\prime}$ applies to $p_{a}$, Case A of the algorithm applies and hence $s^{\prime}$ must be rejected since $M\left(p_{a}\right)$ contains no unpromoted student. If $p_{a}$ is under-subscribed when $s^{\prime}$ applies to $p_{a}$, then some student was already rejected by $p_{a}$. At that time, $\ell_{k}$ must have been full and $p_{a}$ was $\ell_{k}$ 's worst non-empty project. Therefore, $\ell_{k}$ is still full and $p_{a}$ is $\ell_{k}$ 's worst non-empty project or worse than $\ell_{k}$ 's worst non-empty project. Then we can apply the same argument as in Case (2).

Lemma 3.2. Suppose that, during the execution of SPA-P-APPROX-PROMOTION, a project $p_{a}$ has rejected a student. Then after that point, no unpromoted student can be accepted to $p_{a}$. 
Proof. The proof is basically similar to that of the previous lemma, and hence we give only a brief sketch. Let $\ell_{k}$ be the lecturer who offers $p_{a}$. After the rejection point, $\ell_{k}$ or $p_{a}$ is full. If $\ell_{k}$ is full but $p_{a}$ is under-subscribed, then $p_{a}$ must be $\ell_{k}$ 's worst non-empty project or worse than that. Then, afterwards, $\ell_{k}$ has no incentive to accept an unpromoted student to $p_{a}$. Next, suppose that $p_{a}$ is full after the rejection point. As long as $p_{a}$ remains full, $p_{a}$ rejects an unpromoted student. If $p_{a}$ becomes under-subscribed, then $\ell_{k}$ must be full and $p_{a}$ is $\ell_{k}$ 's worst non-empty project or worse than that. Hence we can apply the same argument as the former case.

To prove the stability, we need to prove that there is no coalition or blocking pair.

Lemma 3.3. The output matching $M$ is coalition-free.

Proof. Suppose that there is a coalition $\left\{s_{i_{0}}, s_{i_{1}}, \ldots, s_{i_{r-1}}\right\}$ for some $r \geq 2$. Let $p_{i_{j}}=M\left(s_{i_{j}}\right)$ for each $j(0 \leq j \leq r-1)$. Thus $s_{i_{j}}$ prefers $p_{i_{j+1}}$ to $p_{i_{j}}$ (where $j+1$ is taken modulo $r$ ). Therefore, at some point of the execution, $p_{i_{j+1}}$ was deleted from $s_{i_{j}}$ 's list. Note that during the execution of the algorithm, one project may be deleted from a student's list twice (because of a promotion). Hereafter, a "deletion" means the final deletion unless otherwise stated.

Now suppose without loss of generality that among such deletions, the first occurrence was the deletion of $p_{i_{1}}$ from $s_{i_{0}}$ 's list. First, suppose that $s_{i_{0}}$ is eventually unpromoted. Note that $s_{i_{1}}$ applied to and was accepted by $p_{i_{1}}$ after $s_{i_{0}}$ was rejected by $p_{i_{1}}$. Therefore $s_{i_{1}}$ is eventually promoted by Lemma 3.2. Then $s_{i_{1}}$ was rejected by $p_{i_{2}}$ when $s_{i_{1}}$ was promoted. This means that $s_{i_{2}}$ is eventually promoted by Lemma 3.1(ii). Repeating this argument, we can conclude that $s_{i_{r-1}}$ is eventually promoted. Then this contradicts Lemma 3.1(ii) since $p_{i_{0}}$ rejected the promoted student $s_{i_{r-1}}$ but is assigned an unpromoted student $s_{i_{0}}$ in $M$.

Next suppose that $s_{i_{0}}$ is eventually promoted. Then since $p_{i_{1}}$ rejected a promoted student $s_{i_{0}}$, after that $p_{i_{1}}$ accepts no student by Lemma 3.1(i). This contradicts the fact that $s_{i_{1}}$ was accepted to $p_{i_{1}}$ later.

Lemma 3.4. The output matching $M$ has no blocking pair.

Proof. Assume that there exists a blocking pair $\left(s_{r}, p_{t}\right)$ for $M$. Then it is clear that $s_{r}$ was rejected by $p_{t}$ during the execution (recall that this rejection is the second one if $s_{r}$ was eventually promoted). Let $\ell_{k}$ be the lecturer who 
offers $p_{t}$. Rejections occur at lines $12,14,17,23$, and 25 . If this rejection occurred at line 17,23 , or 25 , then $p_{t}$ was already $\ell_{k}$ 's worst non-empty project or worse than that, and this is also the case in $M$. We know that $\ell_{k}$ was full at this rejection point, and remains full in $M$. Therefore, $\left(s_{r}, p_{t}\right)$ cannot block $M$. If this rejection occurred at line 12 or 14 as a result of $\ell_{k}$ being full and $p_{t}$ being $\ell_{k}$ 's worst non-empty project, then the same argument holds. Therefore suppose that this rejection occurred at line 12 or 14 as a result of $p_{t}$ being full. Since $\left(s_{r}, p_{t}\right)$ blocks $M, p_{t}$ is under-subscribed in $M$. Then $p_{t}$ changed from being full to being under-subscribed at some point. This can happen only when $\ell_{k}$ is full and $p_{t}$ is $\ell_{k}$ 's worst non-empty project. Again, we can use the same argument to show that $\left(s_{r}, p_{t}\right)$ cannot block $M$, a contradiction.

The following lemma follows immediately from Lemmas 3.3 and 3.4.

Lemma 3.5. SPA-P-APPROX-PROMOTION returns a stable matching.

\subsection{Analysis of the Approximation Ratio}

For a given instance $I$, let $M$ be a matching output from SPA-P-APPROXPROMotion, and let $M_{\text {opt }}$ be a largest stable matching for $I$.

Lemma 3.6. $\left|M_{\text {opt }}\right| \leq \frac{3}{2}|M|$.

Proof. Based on $M$ and $M_{o p t}$, we define a bipartite graph $G_{M, M_{o p t}}=$ $(U, V, E)$ as follows: Each vertex in $U$ corresponds to a student in $I$, and each vertex in $V$ corresponds to a position of a project in $I$. Precisely speaking, for each project $p_{j}$ whose capacity is $c_{j}$, we create $c_{j}$ "positions" of $p_{j}$, each of which can accept at most one student, and each vertex in $V$ corresponds to each such position. We use $s_{i}$ to denote the vertex in $U$ corresponding to a student $s_{i}$ and $p_{j, 1}, p_{j, 2}, \ldots, p_{j, c_{j}}$ to denote the vertices in $V$ corresponding to a project $p_{j}$.

If a student $s_{i}$ is assigned to a project $p_{j}$ in $M\left(M_{o p t}\right.$, respectively), we include an edge $\left(s_{i}, p_{j, t}\right)$ for some $t\left(1 \leq t \leq c_{j}\right)$, called an $M$-edge $\left(M_{o p t}\right.$-edge, respectively), in $E$. If $s_{i}$ is assigned to the same project $p_{j}$ both in $M$ and $M_{o p t}$, then $M$ - and $M_{o p t}$-edges corresponding to this assignment include the same position of $p_{j}$, which means we give parallel edges $\left(s_{i}, p_{j, t}\right)$ for some $t$. We also ensure that there are no two vertices $p_{j, t_{1}}$ and $p_{j, t_{2}}$ such that $p_{j, t_{1}}$ is matched in $M$ but not in $M_{o p t}$, and $p_{j, t_{2}}$ is matched in $M_{o p t}$ but not in $M$. In 
such a case, there will be $M$-edge $\left(s_{i_{1}}, p_{j, t_{1}}\right)$ and $M_{\text {opt }}$-edge $\left(s_{i_{2}}, p_{j, t_{2}}\right)$. Then we can remove $\left(s_{i_{1}}, p_{j, t_{1}}\right)$ and add $\left(s_{i_{1}}, p_{j, t_{2}}\right)$ instead.

Note that each vertex of $G_{M, M_{o p t}}$ has degree at most two. Therefore its connected components (other than isolated vertices) are alternating paths or alternating cycles. Now we will modify $G_{M, M_{o p t}}$ while retaining this property and keeping the numbers of $M$-edges and $M_{o p t}$-edges unchanged. Note that the resulting graph may not correspond to a feasible solution for $I$. We use this modification only for the purpose of comparing the sizes of $M$ and $M_{\text {opt }}$.

A connected component consisting of only one $M_{\text {opt }}$-edge is called a Type-I component. A connected component which is a length-three alternating path consisting of two $M_{o p t}$-edges and one $M$-edge in the middle is called a Type-II component. We show that there are no Type-I or Type-II components in the resulting bipartite graph. If this is true, the connected component having the largest ratio of the number of $M_{o p t}$-edges to that of $M$-edges is a length-five alternating path with three $M_{o p t}$-edges and two $M$-edges, which has the ratio of 1.5. This proves the lemma.

Consider a Type-I component $\left(s_{i}, p_{j, t}\right)$. Let $\ell_{k}$ be the lecturer who offers $p_{j}$. Since $p_{j, t}$ is not matched in $M, p_{j}$ is under-subscribed in $M$. Then $\ell_{k}$ must be full in $M$ since otherwise $\left(s_{i}, p_{j}\right)$ blocks $M$. Because $p_{j, t}$ is matched in $M_{o p t}$ but not in $M$, we can find a vertex $p_{a, x}$ in $V$ which is matched in $M$ but not in $M_{o p t}$, where $p_{a}$ is offered by $\ell_{k}$. We can remove $\left(s_{i}, p_{j, t}\right)$ and add $\left(s_{i}, p_{a, x}\right)$ to remove this Type-I component.

Consider a Type-II component $s_{i}-p_{a, x}-s_{j}-p_{b, y}$. Note that $p_{a} \neq p_{b}$ due to the construction of $G_{M, M_{o p t}}$. Since $s_{i}$ is unassigned in $M, s_{i}$ is promoted. Then $s_{i}$ applied to $p_{a}$ when promoted, but was rejected. Therefore $s_{j}$ must be promoted by Lemma 3.1(ii). This means that $s_{j}$ applied to $p_{b}$ at least once, but was rejected. Let $\ell_{k}$ be the lecturer who offers $p_{b}$. As mentioned several times before, this rejection can happen only when (1) $p_{b}$ is full or (2) $\ell_{k}$ is full and $p_{b}$ is $\ell_{k}$ 's worst non-empty project or worse than that, and either (1) or (2) also holds for the output matching $M$. However $p_{b, y}$ is unmatched in $M$, so (2) must hold for $M$ and hence $\ell_{k}$ is full in $M$. Since $\ell_{k}$ is full in $M$ but $p_{b, y}$ is matched only in $M_{o p t}$, there must be a vertex $p_{c, z}$ in $V$ which is matched in $M$ with some vertex, say $s_{d}$, but not matched in $M_{o p t}$, where $p_{c}$ is offered by $\ell_{k}$. If we remove the edge $\left(s_{j}, p_{b, y}\right)$ and add $\left(s_{j}, p_{c, z}\right)$, then we will have an alternating path $s_{i}-p_{a, x}-s_{j}-p_{c, z}-s_{d} \cdots$ of length at least four. Hence this Type-II component is removed.

Note that in both of these cases, we used the property that $\ell_{k}$ is full in $M$. This implies that for each Type-I or Type-II component, we can find 
a distinct vertex in $V$ which is matched only in $M$ to perform the above mentioned replacement. We do this replacement for all Type-I and Type-II components in $G_{M, M_{o p t}}$. This operation does not change any $M$-edges, so the number of students assigned to each lecturer or project in $M$ is unchanged. In particular, a lecturer or a project full in $M$ is still full in the modified graph.

As a result of these operations, we may still have a Type-II component. This can happen only when we removed a Type-I component, such as $\left(s_{i}, p_{j, t}\right)$, using a length-two path, such as $p_{a, x}-s_{r}-p_{b, y}$, where $\left(s_{r}, p_{a, x}\right)$ is an $M$ edge and $\left(s_{r}, p_{b, y}\right)$ is an $M_{o p t}$-edge. In this example, we removed $\left(s_{i}, p_{j, t}\right)$ and added $\left(s_{i}, p_{a, x}\right)$, and as a result we now have a Type-II component $s_{i}-p_{a, x}-$ $s_{r}-p_{b, y}$. Note that $p_{a}$ and $p_{j}$ must be offered by the same lecturer, such as $\ell_{k}$, because of the definition of the operation for Type-I components. Also, by the construction of $G_{M, M_{o p t}}, p_{a}$ and $p_{j}$ must be different projects because $p_{j, t}$ is matched only in $M_{o p t}$ and $p_{a, x}$ is matched only in $M$.

If $p_{b}$ is also offered by $\ell_{k}$, then corresponding to the $M_{o p t}$-edge $\left(s_{r}, p_{b, y}\right)$, we can find a vertex $p_{c, z}$ in $V$ which is matched in $M$ but not in $M_{o p t}$, where $p_{c}$ is offered by $\ell_{k}$, since $\ell_{k}$ is full in $M$. Then we can remove this TypeII component by replacing $\left(s_{r}, p_{b, y}\right)$ with $\left(s_{r}, p_{c, z}\right)$. Otherwise, let $\ell_{k^{\prime}}\left(\neq \ell_{k}\right)$ be the lecturer who offers $p_{b}$. Suppose that $s_{r}$ prefers $p_{b}$ to $p_{a}$. Since $p_{b}$ is under-subscribed in $M, \ell_{k^{\prime}}$ must be full in $M$, since otherwise $\left(s_{r}, p_{b}\right)$ blocks $M$. Then we can use the same argument as before to show the existence of a vertex $p_{c, z}$ which is matched in $M$ but not in $M_{o p t}$, where $p_{c}$ is offered by $\ell_{k^{\prime}}$, and we can replace $\left(s_{r}, p_{b, y}\right)$ with $\left(s_{r}, p_{c, z}\right)$. Suppose that $s_{r}$ prefers $p_{a}$ to $p_{b}$. If $\ell_{k}$ prefers $p_{a}$ to $p_{j}$, then $\left(s_{r}, p_{a}\right)$ blocks $M_{o p t}$, a contradiction (note that $p_{a, x}$ is not matched in $M_{o p t}$ and hence $p_{a}$ is under-subscribed in $M_{o p t}$ ). If $\ell_{k}$ prefers $p_{j}$ to $p_{a}$, then $\left(s_{i}, p_{j}\right)$ blocks $M$, a contradiction. We have exhausted all of the cases, and have shown that all Type-I and Type-II components can be removed. This completes the proof.

The following theorem follows immediately from Lemmas 3.5 and 3.6.

Theorem 3.7. SPA-P-APPROX-PROMOTION is a 1.5-approximation algorithm for $M A X-S P A-P$.

\subsection{Tightness of the Analysis}

We give an instance to show that our analysis of the approximation ratio is tight. There are three students $s_{1}, s_{2}$, and $s_{3}$ and one lecturer $\ell_{1}$ with $d_{1}=3$. 
Lecturer $\ell_{1}$ offers three projects $p_{1}, p_{2}$, and $p_{3}$, where $c_{1}=c_{2}=c_{3}=1$. The preferences of the students and the lecturer are as follows:

$$
\begin{array}{lllllll}
s_{1}: & p_{1} & & \ell_{1}: & p_{3} & p_{2} & p_{1} \\
s_{2}: & p_{1} & p_{2} \\
s_{3}: & p_{2} & p_{3}
\end{array}
$$

Note that the matching $\left\{\left(s_{1}, p_{1}\right),\left(s_{2}, p_{2}\right),\left(s_{3}, p_{3}\right)\right\}$ of size three is stable, but the following execution of SPA-P-APPROX-PROMOTION yields a stable matching of size two $\left\{\left(s_{2}, p_{1}\right),\left(s_{3}, p_{2}\right)\right\}$ :

1. $s_{1}$ applies to $p_{1}$ and is accepted.

2. $s_{3}$ applies to $p_{2}$ and is accepted.

3. $s_{2}$ applies to $p_{1}$ and is rejected.

4. $s_{2}$ applies to $p_{2}$ and is rejected.

5. $s_{2}$ is promoted.

6. $s_{2}$ applies to $p_{1}$ and is accepted; $s_{1}$ is rejected.

7. $s_{1}$ is promoted.

8. $s_{1}$ applies to $p_{1}$ and is rejected.

\subsection{Promoting Many Times}

In the course of SPA-P-APPROX-PROMOTION, each student is promoted at most once. One of the natural extensions is then to let a student be promoted more than once, where a student with more promotions is more preferred (within the same project). Unfortunately, however, we have a simple example to show that this extension does not improve the approximation ratio. There are three students $s_{1}, s_{2}, s_{3}$, three lecturers $\ell_{1}, \ell_{2}, \ell_{3}$, and four projects, $p_{1}$ and $p_{2}$ offered by $\ell_{1}, p_{3}$ offered by $\ell_{2}$, and $p_{4}$ offered by $\ell_{3}$. All of the capacities of lecturers and projects are one. Preference lists are defined as follows:

$\begin{array}{lllll}s_{1}: & p_{2} & \ell_{1}: & p_{1} & p_{2} \\ s_{2}: & p_{3} & p_{1} & \ell_{2}: & p_{3} \\ s_{3}: & p_{3} & p_{4} & \ell_{3}: & p_{4}\end{array}$

First note that $\left\{\left(s_{1}, p_{2}\right),\left(s_{2}, p_{3}\right),\left(s_{3}, p_{4}\right)\right\}$ of size three is a maximum stable matching. Now, consider the following execution of the extended algorithm:

1. $s_{3}$ applies to $p_{3}$ and is accepted.

2. $s_{2}$ applies to $p_{3}$ and is rejected.

3. $s_{2}$ applies to $p_{1}$ and is accepted. 
Then, afterwards, no matter how many times $s_{1}$ is promoted, $s_{1}$ will be rejected by $p_{2}$. Hence the extended algorithm produces a stable matching of size two. Note that this is also another tight example for Section 3.4.

\section{Inapproximability}

We first define the following optimization variant of the stable marriage problem, which we call MAX-SMTI-1T (abbreviation of "Maximum stable marriage problem with ties and incomplete lists with one-sided ties"). In an input, we have sets of men and women. Each man has an acceptable set of women, whom he is willing to be matched with, and has a preference list that orders his acceptable women in a strict order. Similarly, each woman has an acceptable set of men, and has a preference list for them. The women's preference lists may contain ties, meaning that two or more men in the same tie are considered to be of equal preference for her. A matching is a set of disjoint (man, woman)-pairs $(m, w)$ such that $m$ and $w$ are acceptable to each other. If $(m, w)$ is a pair in a matching $M$, we write $M(m)=w$ and $M(w)=m$. For a matching $M$, a (man, woman)-pair $(m, w) \notin M$ is a blocking pair if (i) $m$ and $w$ are acceptable to each other, (ii) either $m$ is unmatched or prefers $w$ to $M(m)$, and (iii) either $w$ is unmatched or prefers $m$ to $M(w)$. A matching that has no blocking pair is stable. MAX-SMTI-1T is the problem of finding a stable matching of maximum size. For an instance $I$ of MAX-SMTI-1T, let OPT $(I)$ be the size of a maximum stable matching for $I$. The following proposition is obtained by letting $p=1 / 3$ in Theorem 3.2 of [4].

Proposition 4.1. [4] For any $\epsilon>0$, if there is a polynomial-time algorithm that, given a MAX-SMTI-1T instance $I$ with $N$ men and $N$ women, distinguishes between the following two cases, then $P=N P$.

(1) $O P T(I) \geq \frac{7 / 3-\epsilon}{3} N$.

(2) $O P T(I)<\frac{19 / 9+\epsilon}{3} N$.

We prove a similar hardness for MAX-SPA-P by a reduction from MAXSMTI-1T. For an instance $I^{\prime}$ of MAX-SPA-P, let $O P T\left(I^{\prime}\right)$ be the size of a maximum stable matching for $I^{\prime}$.

Theorem 4.2. For any $\epsilon>0$, if there is a polynomial-time algorithm that, given a MAX-SPA-P instance $I^{\prime}$ with $N^{\prime}$ students, distinguishes between the following two cases, then $P=N P$. 
(1) $\operatorname{OPT}\left(I^{\prime}\right) \geq \frac{7 / 3-\epsilon}{3} N^{\prime}$
(2) $\operatorname{OPT}\left(I^{\prime}\right)<\frac{19 / 9+\epsilon}{3} N^{\prime}$

Proof. Let $I$ be a MAX-SMTI-1T instance with $N$ men and $N$ women. Without loss of generality, we assume that acceptability is symmetric, i.e., a man $m$ includes a woman $w$ in his list if and only if $w$ includes $m$ in her list. We construct a MAX-SPA-P instance $I^{\prime}$ with $N^{\prime}$ students. Our reduction satisfies conditions (i) $N^{\prime}=N$ and (ii) $\operatorname{OPT}\left(I^{\prime}\right)=O P T(I)$. Then it is not hard to see that Proposition 4.1 implies Theorem 4.2.

For each man $m_{i}$ of $I$, we create a student $s_{i}$ of $I^{\prime}$, and for each woman $w_{j}$ of $I$, we create a lecturer $\ell_{j}$ of $I^{\prime}$. For each woman $w_{j}$, let $T_{j, 1}, T_{j, 2}, \ldots, T_{j, t}$ be the ties in $w_{j}$ 's preference list in the order of preference, where a man not in a tie is considered as a tie of size one. Then, we create projects $p_{j, 1}, p_{j, 2}, \ldots, p_{j, t}$ that are offered by $\ell_{j}$, where $\ell_{j}$ 's preference list includes these projects in this order. Suppose that in $I$, a man $m_{i}$ includes a woman $w_{j}$ at the $d$ th position in his list, and $m_{i}$ is in a tie $T_{j, k}$ of woman $w_{j}$ 's list. Then, in $I^{\prime}$, student $s_{i}$ includes the project $p_{j, k}$ at the $d$ th position of the list. The capacity of each lecturer and each project is one. This completes the reduction. It is not hard to see that the reduction can be done in polynomial time. To illustrate the reduction, we give an example of MAX-SMTI-1T instance $I$ in Fig. 1 and corresponding MAX-SPA-P instance $I^{\prime}$ in Fig. 2. In a woman's list in Fig. 1, men in the same tie are included in parenthesis.

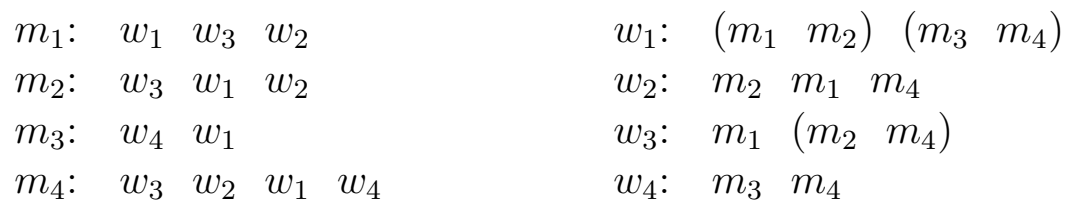

Figure 1: A MAX-SMTI-1T instance $I$

$\begin{array}{lllllllll}s_{1}: & p_{1,1} & p_{3,1} & p_{2,2} & \ell_{1}: & p_{1,1} & p_{1,2} & \\ s_{2}: & p_{3,2} & p_{1,1} & p_{2,1} & \ell_{2}: & p_{2,1} & p_{2,2} & p_{2,3} \\ s_{3}: & p_{4,1} & p_{1,2} & & \ell_{3}: & p_{3,1} & p_{3,2} & \\ s_{4}: & p_{3,2} & p_{2,3} & p_{1,2} & p_{4,2} & \ell_{4}: & p_{4,1} & p_{4,2}\end{array}$

Figure 2: The MAX-SPA-P instance $I^{\prime}$ corresponding to $I$ 
Clearly condition (i) holds. In the rest of the proof, we show that condition (ii) holds. To see this, we show that (A) if there is a stable matching $M$ of $I$, then there is a stable matching $M^{\prime}$ of $I^{\prime}$ such that $\left|M^{\prime}\right|=|M|$, and (B) if there is a stable matching $M^{\prime}$ of $I^{\prime}$, then there is a stable matching $M$ of $I$ such that $|M|=\left|M^{\prime}\right|$. The statement (A) implies $O P T\left(I^{\prime}\right) \geq O P T(I)$ and (B) implies $O P T(I) \geq O P T\left(I^{\prime}\right)$, which together implies condition (ii).

We show (A) first. Given a stable matching $M$ of $I$, we create a matching $M^{\prime}$ of $I^{\prime}$ as follows: Suppose that a man $m_{i}$ is matched with a woman $w_{j}$ in $M$. Then, by construction, $s_{i}$ 's list includes a project $p_{j, k}$ offered by the lecturer $\ell_{j}$, and such $k$ is unique. In $M^{\prime}$, we assign a student $s_{i}$ to $p_{j, k}$. If $m_{i}$ is unmatched in $M$, then $s_{i}$ is unassigned in $M^{\prime}$. It is straightforward to check that $M^{\prime}$ satisfies all of the capacity constraints and that $\left|M^{\prime}\right|=|M|$. Suppose that $M^{\prime}$ admits a blocking pair $\left(s_{i}, p_{j, k}\right)$. Then, $s_{i}$ is unassigned or prefers $p_{j, k}$ to $M^{\prime}\left(s_{i}\right)$. Also, $\ell_{j}$ is unassigned, or assigned a student $s_{i^{\prime}}$ to a project $p_{j, k^{\prime}}$ and $\ell_{j}$ prefers $p_{j, k}$ to $p_{j, k^{\prime}}$. Then, in $M, m_{i}$ is unmatched or prefers $w_{j}$ to $M\left(m_{i}\right)$, and $w_{j}$ is unmatched or prefers $m_{i}$ to $M\left(w_{j}\right)\left(=m_{i^{\prime}}\right)$, i.e., $\left(m_{i}, w_{j}\right)$ is a blocking pair for $M$, contradicting the stability of $M$. Hence, we can conclude that $M^{\prime}$ admits no blocking pair.

Now suppose that $M^{\prime}$ admits a coalition $\left\{s_{i_{0}}, s_{i_{1}}, \ldots, s_{i_{r-1}}\right\}$, that is, $s_{i_{j}}$ prefers $M^{\prime}\left(s_{i_{j+1}}\right)$ to $M^{\prime}\left(s_{i_{j}}\right)$ for each $j$. Then, remove this coalition from $M^{\prime}$, that is, reassign $s_{i_{j}}$ to $M^{\prime}\left(s_{i_{j+1}}\right)$ for each $j$. Note that an application of this operation does not change the matching size. Also, note that no new blocking pair is created because no one becomes worse off. We apply this operation as long as there is a coalition. This sequence of operations must terminate in finite number of steps because at least two students become better off by one application. Hence at the termination, we have a stable matching of size $\left|M^{\prime}\right|$.

Next we show (B). Let $M^{\prime}$ be a stable matching for $I^{\prime}$. We construct a matching $M$ of $I$ as follows: If a student $s_{i}$ is assigned to a project $p_{j, k}$ in $M^{\prime}$, then a man $m_{i}$ is matched with a woman $w_{j}$ in $M$ (note that $w_{j}$ is in $m_{i}$ 's list by construction). If $s_{i}$ is unassigned in $M^{\prime}$, then $m_{i}$ is unmatched in $M$. Again, it is easy to see that $M$ is a stable matching and $|M|=\left|M^{\prime}\right|$. This completes the proof.

Corollary 4.3. Assume that $P \neq N P$. Then for any constant $\delta>0$, there is no polynomial-time $(21 / 19-\delta)$-approximation algorithm for MAX-SPA-P.

Proof. By Theorem 4.2, we see that the existence of a polynomial-time 
algorithm that distinguishes between the following two cases implies $\mathrm{P}=\mathrm{NP}$ for an arbitrary small positive constant $\epsilon$ :

(1) $\operatorname{OPT}\left(I^{\prime}\right) \geq \frac{7 / 3-\epsilon}{3} N^{\prime}$.

(2) $O P T\left(I^{\prime}\right)<\frac{19 / 9+\epsilon}{3} N^{\prime}$.

Suppose that there is a polynomial-time approximation algorithm $T$ for MAX-SPA-P whose approximation ratio is at most $21 / 19-\delta$ for some $\delta$. Then consider these conditions with fixed constant $\epsilon$ such that $\epsilon<\frac{361 \delta}{360-171 \delta}$.

If an instance of Case (1) is given to $T$, it outputs a solution whose size is at least $\frac{7 / 3-\epsilon}{3} N^{\prime} \frac{1}{21 / 19-\delta}$. If an instance of Case (2) is given to $T$, it outputs a solution whose size is less than $\frac{19 / 9+\epsilon}{3} N^{\prime}$. Since $\frac{7 / 3-\epsilon}{3} N^{\prime} \frac{1}{21 / 19-\delta}>\frac{19 / 9+\epsilon}{3} N^{\prime}$ from the definition of $\epsilon, T$ can distinguish between Cases (1) and (2), which implies $\mathrm{P}=\mathrm{NP}$. This completes the proof.

As mentioned in Remark 3.6 of [4], MAX-SMTI-1T is hard to approximate within $1.25-\delta$ for any positive constant $\delta$ if Minimum Vertex Cover problem is hard to approximate within $2-\epsilon$ for any positive constant $\epsilon$ (note that the "if-part" is true if the Unique Games Conjecture is true [6]). Using the reduction in the proof of Theorem 4.2, we can prove the same hardness for MAX-SPA-P:

Theorem 4.4. Assume that, for any positive constant $\epsilon$, there is no polynomialtime $(2-\epsilon)$-approximation algorithm for Minimum Vertex Cover problem. Then, for any positive constant $\delta$, there is no polynomial-time $(1.25-\delta)$ approximation algorithm for $M A X-S P A-P$.

Proof. Suppose that, for some $\delta^{\prime}$, there is a polynomial-time $\left(1.25-\delta^{\prime}\right)$ approximation algorithm $A$ for MAX-SPA-P. Then, the following algorithm $B$ is a polynomial-time $\left(1.25-\delta^{\prime}\right)$-approximation algorithm for MAX-SMTI1T: Given an instance $I$ of MAX-SMTI-1T, $B$ first translates it to an instance $I^{\prime}$ of MAX-SPA-P using the reduction in the proof of Theorem 4.2. It then solves $I^{\prime}$ using $A$ and obtains a solution $M^{\prime}$, and transforms it to a solution $M$ of $I$ in the same manner as given in the proof of Theorem 4.2. Then, by Remark 3.6 of [4], there is a polynomial-time $\left(2-\epsilon^{\prime}\right)$-approximation algorithm for Minimum Vertex Cover problem for some $\epsilon^{\prime}$, which contradicts our assumption. 


\section{Conclusions}

In this paper, we improved the upper and lower bounds on the approximation ratio for MAX-SPA-P. One research direction is to further improve the upper bound. For example, a state of the art approximation algorithm for MAX-SMTI-1T [5] generalizes Király's idea [7] using a Linear Programming approach. Its approximation ratio of $25 / 17(\simeq 1.4706)$ is slightly better than 1.5. One possible next step is to verify whether this idea can be applied to SPA-P-APPROX-PROMOTION.

Acknowledgments. The authors would like to thank the anonymous reviewer for the valuable comments.

\section{References}

[1] D. J. Abraham, R. W. Irving and D. F. Manlove, Two algorithms for the Student-Project Allocation problem, Journal of Discrete Algorithms, 5 (1) (2007) 73-90.

[2] D. Gale and L. S. Shapley, College admissions and the stability of marriage, Amer. Math. Monthly 69 (1962) 9-15.

[3] D. Gusfield and R. W. Irving, The Stable Marriage Problem: Structure and Algorithms, MIT Press, Boston, MA, 1989.

[4] M. M. Halldórsson, K. Iwama, S. Miyazaki, and H. Yanagisawa, "Improved approximation results for the stable marriage problem," ACM Transactions on Algorithms 3 (3) (2007) Article No. 30.

[5] K. Iwama, S. Miyazaki, and H. Yanagisawa, A 25/17-approximation algorithm for the stable marriage problem with one-sided ties, in: Proceedings of ESA 2010: the 18th Annual Europian Symposium on Algorithms, in: Lecture Notes in Computer Science, vol. 6347, SpringerVerlag (2010) 135-146.

[6] S. Khot and O. Regev, Vertex cover might be hard to approximate to within $2-\epsilon$, Journal of Computer and System Sciences 74 (3) (2008) $335-349$.

[7] Z. Király, Better and simpler approximation algorithms for the stable marriage problem, Algorithmica 60 (1) (2011) 3-20. 
[8] D. F. Manlove, and G. O'Malley, Student-project allocation with preferences over projects, Journal of Discrete Algorithms 6 (4) (2008) 553-560. 


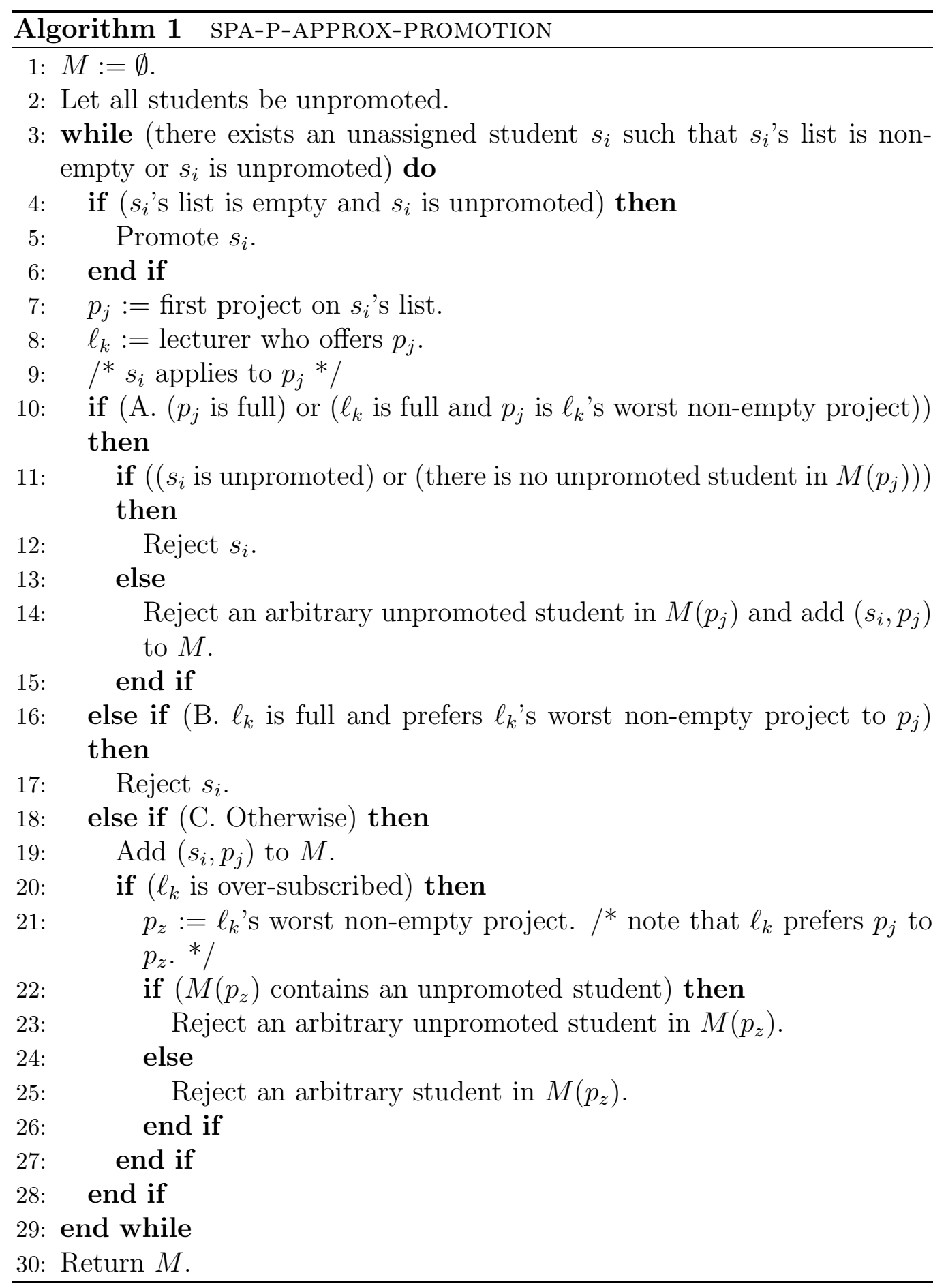

